\title{
Free Flow lon Concentration Polarization Focusing (FF-ICPF)
}

\author{
Vasileios A. Papadimitriou,* Loes I. Segerink, and Jan C. T. Eijkel
}

Cite This: Anal. Chem. 2020, 92, 4866-4874

Read Online

ABSTRACT: Electrokinetic separation techniques in microfluidics are a powerful analytical chemistry tool, although an inherent limitation of microfluidics is their low sample throughput. In this article we report a freeflow variant of an electrokinetic focusing method, namely ion concentration polarization focusing (ICPF). The analytes flow continuously through the system via pressure driven flow while they separate and concentrate perpendicularly to the flow by ICPF. We demonstrate free flow ion concentration polarization focusing (FF-ICPF) in two operating modes, namely peak and plateau modes. Additionally, we showed the separation resolution could be improved by the use of an electrophoretic spacer. We report a concentration factor of 10 in human blood plasma in continuous flow at a flow rate of $15 \mu \mathrm{L} \mathrm{min}{ }^{-1}$.

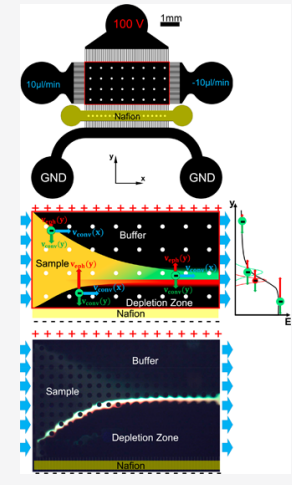

L ab-on-chip (LOC) systems provide an appealing platform for electrophoretic separations due to their high controllability of fluid flow and electric field at the micrometer scale. A subclass of electrophoretic separation methods combines separation and focusing such as electric field gradient focusing, ${ }^{1}$ isoelectric focusing ${ }^{2,3}$ (IEF), and isotachophoresis ${ }^{4,5}$ (ITP). Also, ion concentration polarization focusing (ICPF) belongs to this special class of techniques. In contrast to other techniques of the same class (e.g., ITP and IEF) ICPF does not require any sample preparation or specific electrolytes that can prove to be a tedious process.

Wang et al. ${ }^{6}$ specifically introduced ICPF as a method capable of achieving focusing. They demonstrated extremely high concentration factors in the order of millions. Later Quist et al. ${ }^{7}$ also demonstrated separation of anionic analytes in a similar system. Since its introduction, additional research has been performed with this technique ${ }^{7-12}$ including applications with human blood plasma. ${ }^{13-15}$ Though ICPF offers a very powerful analytical tool, an inherent disadvantage of miniaturizing any separation technique in LOC systems is the low throughput of sample. Additionally, in many cases the separated analytes are effectively trapped in chip, thereby prohibiting any downstream analysis such as by mass spectrometry.

In order to overcome this limitation, a "free flow" variant of a separation method can be used. The term "free flow" refers to methods where the separation direction is perpendicular to the flow direction allowing continuous high-throughput separation and extraction. Several electrophoretic separation methods have been adapted and demonstrated to free flow variants ${ }^{16,17}$ including focusing techniques such as ITP $^{18}$ and IEF, ${ }^{19,20}$ as reviewed by Kohlheyer et al. ${ }^{21}$ Attempts to extract preconcentrated analytes have also been reported, but the use of Quake valves $^{22}$ or magnetically actuated valves ${ }^{23}$ makes the extraction noncontinuous and low throughput (i.e., flow rates in the $\mathrm{nL}$ $\mathrm{min}^{-1}$ range $\left.{ }^{24}\right)$. Continuous nonselective low throughput extraction was also demonstrated in ICPF. ${ }^{25-27}$ ICPF has been used in high throughput applications for separation particles $\left(1 \mu \mathrm{L} \mathrm{min}^{-1}\right),{ }^{28}$ bacterial lysis $\left(>1 \mathrm{~mL} \mathrm{~min}^{-1}\right),{ }^{29}$ and desalination $\left(<20 \mu \mathrm{L} \mathrm{min}^{-1}\right){ }^{30}$ The required flow rate (throughput) in microfluidics ranges widely based on the specific application. In sample preparation applications high throughput is needed; for example in the separation of circulating tumor cells flow rates in the range of $100 \mu \mathrm{L} \mathrm{min}^{-1}$ are required. ${ }^{31,32}$ For the purification of ionic radioisotopes used for medical imaging flow rates ranging between 300 and $1000 \mu \mathrm{L}$ $\min ^{-133}$ have been demonstrated. When it comes to the detection of low abundance analytes such as DNA and proteins, however, the required flow rates are much lower, ranging from 1 $\mu \mathrm{L} \min ^{-1}$ to $2.5 \mu \mathrm{L} \mathrm{min}{ }^{-1} .^{34-36}$ For electrokinetic free flow variants flow rates up to $20 \mu \mathrm{L} \mathrm{min}{ }^{-137}$ have been reported but that included the flow of the sample and the flow of the buffers that are required in such techniques. In addition, the throughput is determined by the sensitivity of the detection method and the concentration of the analyte of interest present in the sample. ${ }^{38}$ In this article we propose a setup for continuous high throughput concentration and separation of anionic analytes based on free flow ICPF (FF-ICPF).

Received: October 4, 2019

Accepted: March 20, 2020

Published: March 20, 2020 


\section{THEORY}

A schematic of the FF-ICPF process is shown in Figure 1. The microfluidic device consists of a chamber where the separation takes place, which is connected via a Nafion patterned region to a microchannel. An electric field is applied in the $y$ direction and a pressure-driven flow (PDF) perpendicular to the electric field ( $x$ direction, Figure 1). We will first give a short description of ICPF in order to understand the dynamics of our system. ICPF is a concentration and separation method of ionic species based on their differential migration in an electric field gradient. In ICPF the electric field gradient is created via a constant electric potential across a background electrolyte concentration gradient. The concentration gradient is thereby created via the phenomenon of ion concentration polarization (ICP). ${ }^{39}$ When a potential is applied across a cation perm-selective membrane, the biggest fraction of the current is carried by the cations. The flux imbalance between cations and anions through the permselective region removes anions from the anodic side resulting in a zone with low concentration of all species known as the depletion zone. Such a cation perm-selective zone can be a nanochannel $^{39}$ or a cation perm-selective polymer (e.g., Nafion ${ }^{11}$ ).

The requirement of a constant current density in all regions (due to charge conservation) dictates that the application of a constant potential across the separation chamber will result in a high electric field in the depletion zone (low conductivity), a low

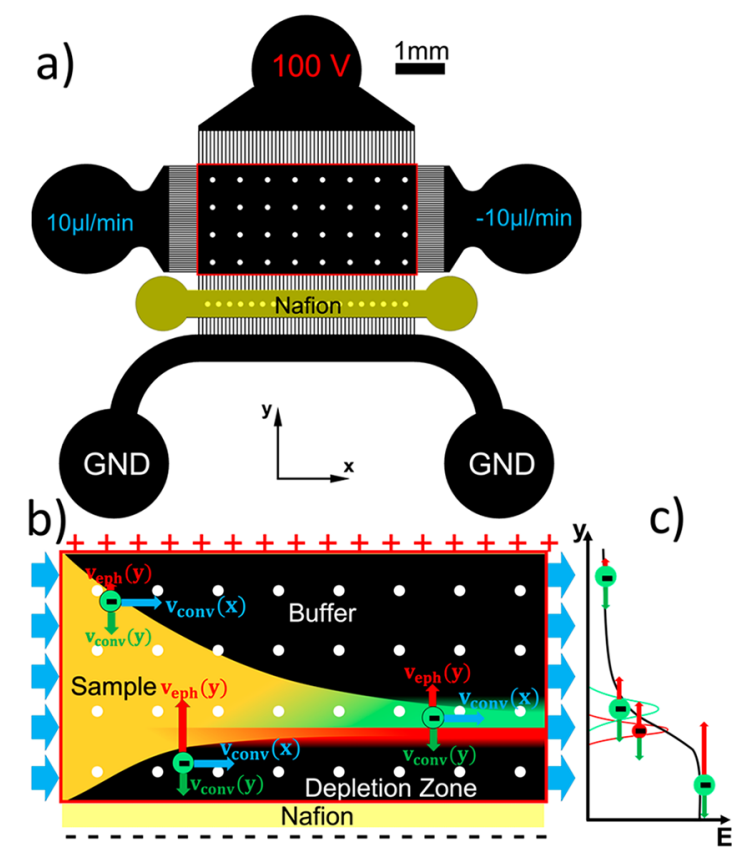

Figure 1. (a) Design of the device and a typical actuation scheme. (b) Schematic of operation principle of FF-ICPF. The sample enters and flows across the separation chamber by PDF (main contribution to $v_{\text {conv }}(x)$ ) and an E-field is applied perpendicular to the flow (in contrast to simple ICPF where the E-field and flow have the same direction). (c) The focusing mechanism of ICPF in the $y$ direction. An electric field gradient is created due to the ion depletion zone. The analytes focus at the $y$ position where the EOF (main contribution to $v_{\text {conv }}(y)$ ) and electrophoretic flow $\left(v_{\text {eph }}(y)\right)$ are equal and opposite. A different analyte with a different electrophoretic mobility requires a different electric field to acquire the same $v_{\text {eph }}(y)$ hence it will focus at a different position in the E-field gradient (present between depletion zone and bulk solution). electric field in the bulk region (high conductivity) and an electric field gradient in between. The fluxes of ions are described via the Nernst-Planck equation as the sum of the diffusive, convective and electrophoretic flux. If we neglect diffusion for simplicity, we can write the total flux of analyte ion $i$, $\mathbf{J}_{i}\left[\mathrm{~mol} \mathrm{~m}^{-2} \mathrm{~s}^{-1}\right]$ as follows:

$$
\begin{aligned}
& \mathbf{J}_{i}=\mathbf{J}_{i, \mathrm{conv}}+\mathbf{J}_{i, \mathrm{eph}} \\
& \mathbf{J}_{i}=v_{\text {conv }} C_{i}+v_{i, \mathrm{eph}} C_{i}
\end{aligned}
$$

Here $\mathbf{J}_{i, \text { conv }}$ and $\mathbf{J}_{i, \text { eph }}$ are the convective and electrophoretic fluxes, $v_{\text {conv }}\left[\mathrm{m} \mathrm{s}^{-1}\right]$ is the linear convective bulk velocity, and $v_{i, \text { eph }}\left[\mathrm{m} \mathrm{s}^{-1}\right]$ and $C_{i}\left[\mathrm{~mol} \mathrm{~m}^{-3}\right]$ are the electrophoretic velocity and concentration of species ${ }_{i}$, respectively.

We are now going to investigate the velocity contributions separately in the $x$ - and $y$ directions (see Figure 1).

Convection. The convective velocity is a combination of a $\mathrm{PDF}$ and electroosmotic flow (EOF). The PDF is applied via two syringe pumps; one is pushing liquid through the inlet and the other is sucking liquid from the outlet with the same flow rate. The use of two syringe pumps in combination with the high hydraulic resistance of the channels connected to the chamber in the vertical direction ensures a uniform horizontal PDF $\left(v_{\text {conv }, \mathrm{x}}=\right.$ constant). The equivalent hydraulic resistance of all the vertical channels is approximately 5 times higher than the total resistance of the chamber in the $x$ direction.

The electroosmotic velocity follows the electric field according to the Helmholtz-Smoluchowski equation:

$$
v_{\mathrm{EOF}}=\frac{\varepsilon \varepsilon_{0} \zeta}{\eta} E
$$

Here $\eta\left[\mathrm{kg} \mathrm{m}^{-1} \mathrm{~s}^{-1}\right]$ is the viscosity of the liquid, $\zeta[\mathrm{V}]$ is the zeta potential, $\varepsilon$ and $\varepsilon_{0}\left[\mathrm{~F} \mathrm{~m}^{-1}\right]$ are the relative and vacuum permittivity and $E\left[\mathrm{~V} \mathrm{~m}^{-1}\right]$ is the electric field. A high positive potential is applied to the top reservoir (Figure 1) and the bottom reservoirs are grounded. The high electric resistance (12 times higher) of the channel array connecting the left- and righthand reservoirs to the chamber (in the horizontal direction in Figure 1) ensures that only a small fraction of the current will flow through them. Hence, we assume that the electric field direction points along the $y$ direction from top to bottom in the separation chamber $\left(E_{x}=0\right)$ ). The electric field is not uniform along the $y$ direction due to the depletion zone that forms as described above. As a result, as described by eq 3, the electroosmotic velocity in the $y$ direction is not uniform. Due to the incompressibility of aqueous electrolytes and because of mass conservation, a negative pressure is induced at the location of the electric field gradient. ${ }^{24}$ The hereby induced PDF creates a constant and uniform flow in the $y$ direction $\left(v_{\text {conv, } \mathrm{x}}=\right.$ constant). EOF-induced pressures have been previously reported at electric field gradients ${ }^{40-42}$ or in channels with nonuniform zeta potential. ${ }^{43}$ In reality the total convective flow due to the electric field in ICPF is enhanced and higher than the EOF as demonstrated by Kim et al. ${ }^{8}$ It is worth mentioning that the Nafion membrane does not hydrodynamically close the system, since it does not completely block the channel. The Nafion membrane thus resembles the one in the system reported by Ko et al., ${ }^{11}$ where they demonstrated ICPF in a microchannel where only the bottom was patterned with a strip of Nafion. Despite the convective flow on top of the Nafion, the cationic flux through the Nafion was much higher than in the bulk and sufficient to create the ICP phenomenon. ${ }^{11}$ 
Electroconvective vortices are formed close to an ion selective membrane when they are operated in the overlimiting current regime. ${ }^{44}$ These vortices are growing in size as the potential increases and eventually result in fluidic instabilities (chaotic depletion zone patterns). ${ }^{45-48}$ Such fluidic instabilities can disrupt the ICPF process. In our system no chaotic depletion zone patterns (i.e., no disruption of the focusing process) were noticed for potentials up to $700 \mathrm{~V}$. In addition, the Nafion-filled small microchannels resemble the approach of Kim et al., ${ }^{49}$ who report the reduction of electroconvective vortices by the use of "microfins".

Analyte Electrophoresis. Anions will migrate in the direction opposite to the electric field and the electrophoretic velocity $v\left({ }_{i, \mathrm{eph}}\right)$ of anion $i$ scales linearly with the electric field

$$
v_{i, \mathrm{eph}}=\mu_{i} E
$$

where $\mu_{i}\left[\mathrm{~m}^{2} \mathrm{~s}^{-1} \mathrm{~V}^{-1}\right]$ is the electrophoretic mobility of species $i$. As described earlier we assume no electric field in the $x$ direction and, hence, no electrophoresis in that direction.

Analyte Focusing. We first summarize the velocities in the $x$ and $y$ directions for an anionic species $i$

$$
\begin{aligned}
& v_{x, i}=v_{x, \mathrm{conv}}+v_{x, i, \mathrm{eph}} v_{y, i}=v_{y, \mathrm{conv}}+v_{y, i, \mathrm{eph}} \\
& v_{x, \mathrm{conv}}=\text { constant }, \quad v_{x, i, \mathrm{eph}}=0, \quad v_{y, \mathrm{conv}}=\text { constant } \\
& v_{y, i, \mathrm{eph}}=\mu_{i} E_{y}
\end{aligned}
$$

An anion will be transported in the $x$ direction with a constant velocity in the separation chamber, as is used for the continuous extraction of the focused analytes. The focusing of the analytes occurs in the $y$ direction. In the $y$ direction the velocity of the anion is the sum of a constant convective velocity and an opposite, electric field-dependent electrophoretic velocity. As the field is nonuniform in the $y$ direction, the total velocity depends on the location in the electric field gradient. If the anion is far away from the depletion zone (the buffer zone in Figure 1) the electric field is low and $v_{y, \text { conv }}$ is the dominant contribution, moving the anion toward the depletion zone. If the anion is in the depletion zone where the electric field is the highest, electrophoresis dominates and migrates the anion upward toward the buffer zone. At some location in the electric field gradient between bulk and depletion zone, the $y$ contributions of convective and electrophoretic velocities are equal and opposite resulting in a zero net $y$ direction velocity and hence focusing of the anion. A different anion with a different electrophoretic mobility requires a different electric field to acquire a zero net velocity and, hence, will focus at a different location in the electric field gradient.

Operation Modes. In many focusing techniques, such as $\operatorname{ITP}^{7,50}$ two distinct operation modes can be found, namely peak mode and plateau mode. In peak mode the analytes are in very low concentration compared to the background electrolyte so we assume that they do not contribute to the local conductivity of the solution. Hence the analytes do not affect the local electric field and the concentration process. This is the most common operation mode of focusing methods where the main goal is the concentration of low abundance species. In peak mode the analytes are concentrating and forming a Gaussian concentration profile (in the separation direction). The concentration profile of analytes in separation sciences is a long and well investigated topic. ${ }^{51-53}$ As described in previous work ${ }^{24}$ the variance $\left.\left(\sigma_{i}^{2}\left[\mathrm{~m}^{2}\right]\right)\right)$ of the Gaussian profile of a species $i$ is given by

$$
\sigma_{i}^{2}=\frac{V_{\mathrm{T}}}{z_{i} \frac{\mathrm{d} E_{y}}{\mathrm{~d} y}}
$$

where $V_{\mathrm{T}}[\mathrm{V}]$ is the thermal potential $\left(\left(V_{\mathrm{T}}=k_{\mathrm{b}} T / e\right.\right.$ with $T[\mathrm{~K}]$ the temperature, $k_{\mathrm{b}}\left[\mathrm{J} \mathrm{K}^{-1}\right]$ Boltzmann's constant, and $e[\mathrm{C}]$ the elementary charge), $z_{i}$ is the valence of the species $i$, and $\mathrm{d} E_{y} / \mathrm{d} x$ $\left[\mathrm{V} \mathrm{m}^{-2}\right]$ is the electric field gradient between the bulk and depletion zone in the $y$ direction. We assume that the electric field gradient is constant. In addition, the separation resolution $R_{\mathrm{s}}$ for two species can be calculated as

$$
\begin{aligned}
& R_{\mathrm{s}}=\frac{d_{x}}{2\left(\sigma_{1}+\sigma_{2}\right)} \\
& R_{\mathrm{s}}=\frac{v_{y, \text { conv }}}{2 \sqrt{\frac{\mathrm{d} E_{y}}{\mathrm{~d} y} V_{\mathrm{T}}}} \cdot \frac{\left(\frac{1}{\mu_{1}}-\frac{1}{\mu_{2}}\right)}{\left(\frac{1}{\sqrt{z_{1}}}+\frac{1}{\sqrt{z_{2}}}\right)}
\end{aligned}
$$

Here $\sigma_{1}[\mathrm{~m}]$ and $\sigma_{2}[\mathrm{~m}]$ are the standard deviations of the analyte Gaussian peaks and $d_{x}[\mathrm{~m}]$ is the distance between the peaks (i.e., the difference of the mean value). In eq $8 \mathrm{~b}$ the first term is dependent on the applied potential and device geometry and the second term is dependent on the analyte properties.

In contrast, if the concentrated analyte approaches the background electrolyte concentration, its contribution to the conductivity and electric field can no longer be neglected. In this case the analyte is in plateau mode. As indicated by the name, the analyte will concentrate until a maximum concentration is reached and a plateau is formed in the concentration profile which will then widen over time. The constant concentration of the plateau locally creates a constant electric field. A known analyte in plateau mode (of which the electrophoretic mobility is between two analytes of interest) can be used as an electrophoretic spacer to push these two analytes apart and thereby improving their separation resolution. Electrophoretic spacers have been reported before in various focusing techniques, ${ }^{5,50,51}$ including ICPF. ${ }^{12,54}$

In standard ICPF, there is a continuous supply of analytes toward their focusing location. Therefore, an extremely high concentration factor can be achieved until the analyte reaches plateau mode. Quist et al. ${ }^{12}$ report concentration factors up to $10^{7}$ using ICPF, and recently concentration factors in the order of billions were reported. ${ }^{14}$ The theoretical limit of enrichment in peak mode was expressed by Ouyang et al., ${ }^{41}$ who showed it depends on the mobilities of the analytes and the background electrolyte co-ion as well as on the Peclet number of the system. In FF-ICPF there is no continuous supply of analyte in the separation direction, hence the maximum concentration is limited by the length of the chamber in the $y$ direction. As the analytes move along the chamber (in the $x$ direction), they are getting "squeezed" down to either a Gaussian profile in peak mode or a plateau in the plateau mode. When the analytes reach peak mode, the maximum concentration can be calculated by the ratio of the chamber width $(W[\mathrm{~m}])$ compared to the standard deviation of the Gaussian peak.

$$
C_{\text {max,peak }}=\frac{W}{\sigma_{i} \sqrt{2 \pi}} C_{\text {bulk }}
$$

given that the area $\left(C_{A}\left[\mathrm{~m}^{-2}\right]\right)$ under a Gaussian peak equals $C_{\mathrm{A}}=C_{\text {max,peak }} \sigma_{i} \sqrt{2 \pi}$. In the case of plateau mode, the plateau concentration can be calculated from the Kohlrausch regulation 
function (KRF). ${ }^{55}$ The KRF is a conservation law (conservation function) that is derived by the continuity equations describing the electromigration in an electric field. The KRF is mainly used in electrophoresis which enables the calculations of the adjusted concentration of constituents. ${ }^{56}$ The KRF requires the KRF value (eq 10) to be constant over time in all regions (bulk, depletion zone, and any plateau mode zones).

$$
\operatorname{KRF}(y)=\sum_{i} \frac{z_{i} C_{i}(y, t)}{\mu_{i}}
$$

Throughput. The throughput of the device is determined by the volumetric flow rate $\left(Q_{s}\left[\mathrm{~m}^{3} \mathrm{~s}^{-1}\right)\right)$ of the sample. If we assume that the PDF applied by the syringe pump is the only contribution for flow in the $x$ direction (i.e., throughput), the time $t_{x}[\mathrm{~s}]$, it takes for an analyte to cross the length of chamber $\left(L_{x}[\mathrm{~m}]\right)$ may be written as

$$
t_{x}=\frac{L_{x} L_{y} h}{Q_{s}}
$$

where $L_{y}[\mathrm{~m}]$ is the length of the chamber in the $y$ direction and $h$ $[\mathrm{m}]$ is the height of the chamber. $t_{x}$ is also the time that the analytes are able to "interact" with the depletion zone and focus. Compared to previous works ${ }^{26,27}$ where only a local depletion zone exists in a microchannel, the interaction time in our device is much longer allowing higher throughput. In order for the analytes to separate, they need to travel to their focusing position. If we take the worst case where the focusing position is at the bottom of the chamber adjacent to the Nafion membrane and an analyte is at the top of the chamber, it needs to travel the entire length of the chamber in the $y$ direction $\left(L_{y}\right)$. If for simplicity we assume that the only contribution to $v_{\text {conv, } y}\left[\mathrm{~m} \mathrm{~s}^{-1}\right]$ is the electroosmotic flow using eq 3 , we can calculate the time $\left(t_{y}[s]\right)$ that an analyte needs to travel through the chamber in the $y$ direction.

$$
t_{y}=\frac{L_{y} \eta}{\varepsilon \varepsilon_{0} \zeta \mathrm{E}_{y}}
$$

In reality $t_{y}$ will be smaller since an enhanced convective flow has been reported in $\mathrm{ICPF}^{8,41}$ due to a secondary induced EOF in the depletion zone. In all cases, $t_{y}$ should be smaller than $t_{x}$ when focusing is to take place. If we equate the two, we can derive a relation between the two means of actuation (by PDF and electric field) needed to satisfy the condition of proper focusing:

$$
Q_{\mathrm{s}}=\frac{\varepsilon \varepsilon_{0} \zeta L_{x} h}{\eta} E_{y}
$$

Once again assuming that there are no effects due to ICPF influencing the electric field, we can state $E_{y}=V / L_{y}$ where $V[\mathrm{~V}]$ is the applied potential across the chamber, and we obtain

$$
Q_{\mathrm{s}}=\frac{\varepsilon \varepsilon_{0} \zeta L_{x} h}{\eta L_{y}} V
$$

In this simplified scaling law, the applied potential is linearly related to the throughput for a specific device. When it comes to device design parameters, the larger $L_{x}$ and $h$, the higher the throughput for a specific potential, since these parameters increase $t_{x}$. In contrast, the larger $L_{y}$, the lower the throughput because of the increase in $t_{y}$. As mentioned before, eq 14 is a simplified scaling law in order to understand how the different actuation and geometric parameters affect the throughput rather than a precise calculation of the throughput. There also is a limit to its applicability, (i) there is a maximum applied electric field before Joule heating will disrupt the process; (ii) since the Nafion is patterned via capillary forces there is a maximum $L_{y}$ for the capillary filling. For longer chambers a different method for the patterning of the ion selective membrane must be used (e.g., a stamping method for planar Nafion membranes has been widely reported ${ }^{57}$ ); (iii) the Nafion is currently patterned on the bottom of the chip and the depletion zone must extend to the whole height of the chamber for the functionality of ICPF, hence the height of the chamber is also limited. In addition, an increased chamber height may increase the impact of the electroconvective vortices in the depletion zone and the overall stability of the system. In the Supporting Information (SI), we furthermore calculate the effect of electric power on the stability of the system. A high electric power can affect the stability in two ways: in the form of heat and in the form of changes in $\mathrm{pH}$ due to electrochemical reactions in the reservoirs.

\section{EXPERIMENTAL SECTION}

In order to characterize our proposed method, microfluidic chips were fabricated out of polydimethylsiloxane (PDMS, DOWSIL 184 Silicone Elastomer kit; 1:10 cross-linker to polymer ratio). A mold was used for standard soft lithography ${ }^{58}$ of the PDMS chips, which were bonded on standard microscopy glass slides after $45 \mathrm{~s}$ of $\mathrm{O}_{2}$ plasma treatment (in a FemtoScience Cute device). The mold was made out of a silicon wafer and 35 $\mu \mathrm{m}$ (height of the chamber $h$ ) of MicroChem SU-8 2050 negative photoresist which was patterned using a photolithography mask (Figure 1a). The size of the separation chamber was $4 \mathrm{~mm}\left(L_{x}\right)$ by $2 \mathrm{~mm}\left(L_{y}\right)$. There are 50 small channels in each side of the chamber. The width and length of the horizontal channels are 5 and $550 \mu \mathrm{m}$, respectively, while the vertical ones are 10 and $750 \mu \mathrm{m}$. The supplier's instructions were followed for the exposure and development of the photoresist.

A small amount (approximately $5 \mu \mathrm{L}$ ) of Nafion perfluorinated resin solution ( $20 \mathrm{wt} \%$, Sigma-Aldrich) was introduced to one of the reservoirs (marked with yellow in Figure 1). The Nafion solution was filled and patterned via capillary forces. ${ }^{9,59}$ Nafion was dried at $60{ }^{\circ} \mathrm{C}$ for $30 \mathrm{~min}$ to form a solid permselective polymer. Nafion resin solution experiences significant shrinkage during the drying process due to solvent evaporation. The shrinkage causes the solid Nafion to detach from the channel walls creating gaps which allow hydrodynamic flow around the solid Nafion.

$\mathrm{An}_{2}$ plasma treatment preceded the testing of each chip to ensure high hydrophilicity of the PDMS walls to avoid any bubble formation during the filling process. The anodic and cathodic reservoirs of the microfluidic chip were filled with a buffer solution (dilutions of phosphate buffered saline (PBS) (Sigma-Aldrich)). The inlet and outlet reservoirs were connected to a NEMESIS syringe pump (two 4605 dosing units, Cetoni Gmbh). Platinum wires (Alfa Aesar 0.01 in. in diameter) were introduced to the anodic and cathodic reservoirs and connected to a Keithley 2410 source meter power supply. For the fluorescent microscopy, an Olympus IX51 was used and images/videos were captured with a FLIR Grasshopper3 color camera. The syringe pump and power supply were operated via neMESYS UI (Cetoni Gmbh) and an in-house made LabVIEW program, respectively. The results were analyzed with Image (V. 1.51) and Matlab (R2016a).

As sample, PBS dilutions or human blood plasma spiked with fluorescent anionic markers were used. Whole human blood 
(with $3.2 \%$ sodium acetate) was provided by the Experimental Centre for Technical Medicine (ECTM, Technical Medical Centre, University of Twente). The whole blood was centrifuged (within $4 \mathrm{~h}$ after donation) for $15 \mathrm{~min}$ at $500 \mathrm{~g}$ in an Allegra X-12R Centrifuge (Beckman Coulter), and the blood plasma was extracted, aliquoted, and stored at $-80^{\circ} \mathrm{C}$. As model anion analytes BODIPY 492/515 Disulfonate (BDP; Invitrogen), Alexa Fluor 647 Carboxylic Acid, tris(triethylammonium) (AF647; Invitrogen) salt, fluorescein sodium salt (SigmaAldrich), and Cascade Blue hydrazide trisodium salt (CB; Invitrogen) were chosen.

\section{RESULTS AND DISCUSSION}

Peak Mode. In peak mode the analytes are in very low concentration compared to the background electrolyte so their contribution to the conductivity can be neglected, and they will form a Gaussian concentration peak during the focusing. We prepared our model sample of $70 \mu \mathrm{M}$ BDP, AF647 and CB in $0.1 \times$ PBS. Our model analytes are approximately 3 orders of magnitude lower in concentration than the dominant ions (15.7 $\mathrm{mM} \mathrm{Na}^{+}, 14.0 \mathrm{mM} \mathrm{Cl}^{-}, 1.0 \mathrm{mM} \mathrm{HPO}_{4}{ }^{2-}$ and $0.2 \mathrm{mM} \mathrm{H}_{2} \mathrm{PO}_{4}{ }^{-}$) in $0.1 \times$ PBS. Our sample was flowed through the chip with a flow rate of $5 \mu \mathrm{L} \mathrm{min}$ minal a $^{-1}$ and a potential of Fluorescent microscopy images were taken after approximately $3 \mathrm{~min}$ as shown in Figure 2. A depletion zone was formed on the anodic side of the Nafion, and the analytes were focused at the region between bulk and depletion zone as they flowed from left to right due to the PDF and from top to bottom due to EOF. As

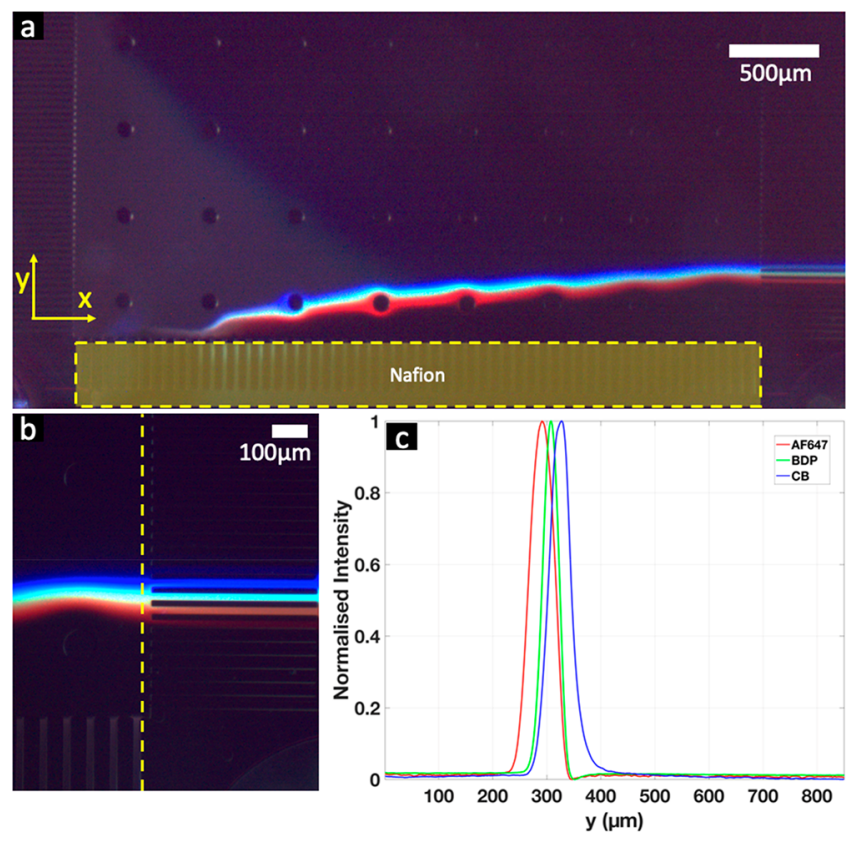

Figure 2. (a) Fluorescent microscopy image of FF-ICPF in 0.1xPBS. Focused streams of CB, BDP and AF647 can be seen $\left(5 \mu \mathrm{L} \mathrm{min}^{-1}\right.$ at 200 V). Nafion is marked with the yellow box. (b) Close-up fluorescent microscopy image of the extracted streams. (c) Normalized fluorescence intensity profiles of CB, BDP, and AF647 (along the yellow line of image b with $y=0$ at the Nafion membrane), the maximum intensity is approximately 17 times higher than the bulk intensity for all three dyes. The fluorescent intensity is normalized independently for each color. Note: The contrast and color balance of microscopy images $a$ and $b$ have been altered for better visibility. The fluorescent intensity profiles have been extracted from the original images (which can be found in the SI, Figure ESI1). shown in Figure 2c, the analytes formed focused streams with a Gaussian concentration profile in the $y$ direction. The added analyte $\mathrm{CB}$ has the highest electrophoretic mobility, followed by BDP and AF647. ${ }^{60}$ This means that AF647, with the lowest mobility, focused at a region of higher E-field (closer to the depletion zone) compared to BDP and CB. Similarly, the CB with the highest mobility focused at a lower E-field compared to BDP. Despite the high concentration of the focused analytes, a clear overlap of the three focused streams can be seen (Figure $2 c)$. Due to the very high electric field gradients in the ICPF, an inherent disadvantage of the method is the poor resolution in peak mode (eq $8 \mathrm{a}$ ). In the example shown in Figure $2 \mathrm{c}$, the separation resolution between AF647 (red) and CB (blue) is $0.26\left(\sigma_{\mathrm{AF} 647}=27 \mu \mathrm{m}, \sigma_{\mathrm{CB}}=29 \mu \mathrm{m}\right.$ and distance between peaks was $30 \mu \mathrm{m}$ as calculate from Figure $2 \mathrm{c}$ ).

Plateau Mode. In order to demonstrate the plateau mode, $0.1 \times$ PBS was spiked with $1 \mathrm{mM}$ of $\mathrm{CB}, \mathrm{AF} 647$, and BDP. In this solution the analytes have approximately a 10 times lower concentration than the background electrolyte. As shown in Figure 3, once the analytes concentrate to their maximum allowed concentrations (eq 10), they form wide plateaus instead of Gaussian concentration profiles.
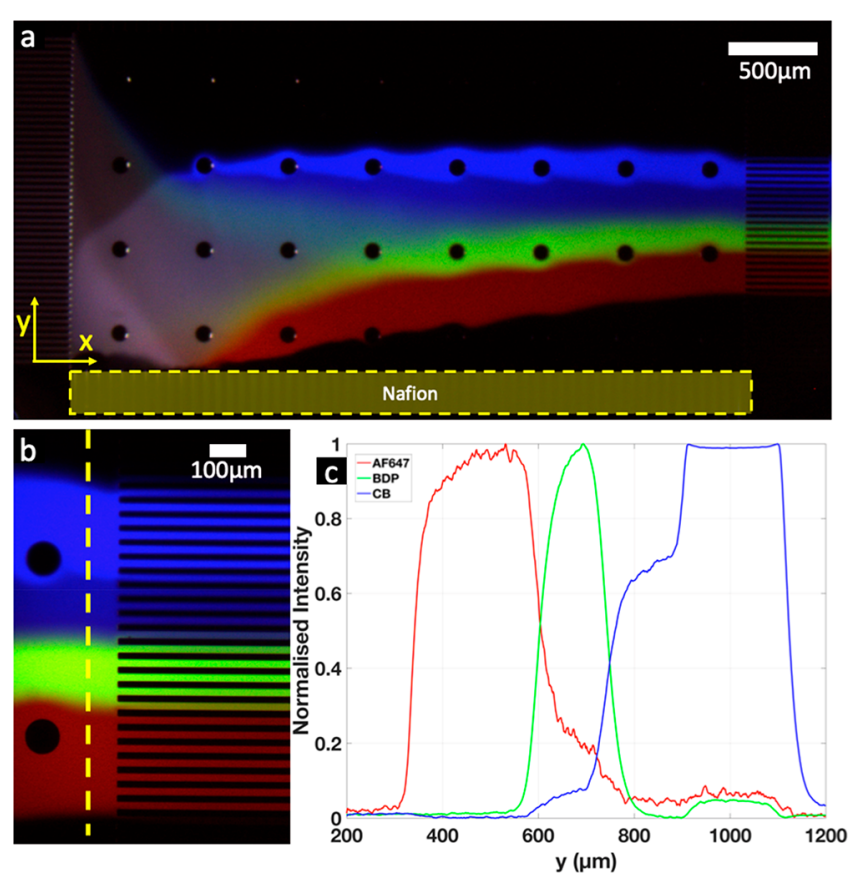

Figure 3. (a) Fluorescent microscopy image of FF-ICPF in $0.1 \times$ PBS. Focused streams of CB, BDP and AF647 can be seen $\left(5 \mu \mathrm{L} \mathrm{min}^{-1}\right.$ at 200 V). Nafion is marked with the yellow box. (b) Close-up fluorescent microscopy image of the extracted streams. (c) Normalized fluorescence intensity profiles of $\mathrm{CB}, \mathrm{BDP}$ and AF647 (along the yellow line of image $\mathrm{b}$ with $y=0$ at the Nafion membrane), the maximum intensity is approximately 4-5 times higher than the bulk intensity for all three dyes. Nevertheless, because of the high starting concentration of the fluorescent dyes the fluorescent intensity is no longer linearly related to concentration of the analyte due to selfquenching. The analytes create plateaus instead of Gaussian peaks. The fluorescent intensity is normalized independently for each color. Note: the contrast and color balance of microscopy images $a$ and $b$ has been altered for better visibility. The fluorescent intensity profiles have been extracted from the original images (which can be found in the SI, Figure ESI2). 
In Figure 3 two different plateaus of blue can be seen. This can be attributed to another species being present between the blue and the green analyte. As the phosphates $\left(\mathrm{HPO}_{4}{ }^{2-}\right.$ and $\mathrm{H}_{2} \mathrm{PO}_{4}^{-}$) are present in sufficiently high concentration to act as spacers, we need to calculate the mobilities of all of the species to see whether they can be functioning as spacer. For this purpose, we used the Einstein-Smoluchowski equation of diffusion, using diffusion coefficients that were either found in the literature or calculated by the formula given by Evans et al. ${ }^{61}$ With the phosphates $\left(\mathrm{HPO}_{4}{ }^{2-}\right.$ and $\left.\mathrm{H}_{2} \mathrm{PO}_{4}{ }^{-}\right)$, electrophoretic mobilities of $5.874 \times 10^{-8}$ and $3.711 \times 10^{-8} \mathrm{~m}^{2} \mathrm{~V}^{-1} \mathrm{~s}^{-1}$ were found, respectively. ${ }^{62}$ Accounting for their concentrations at $\mathrm{pH}$ 7.4 in PBS, the effective mobility of the phosphate couple is $5.544 \times 10^{-8} \mathrm{~m}^{2} \mathrm{~V}^{-1} \mathrm{~s}^{-1}$. Calculated by the same method, in PBS ( $\mathrm{pH}$ 7.4) the mobility of cascade blue $\mathrm{CB}^{3-}$ is $4.32 \times 10^{-8} \mathrm{~m}^{2} \mathrm{~V}^{-1}$ $\mathrm{s}^{-1}$. This means that at $\mathrm{pH} 7.4$ the phosphates will be leading the $\mathrm{CB}$ and will not act as a spacer. In addition, if phosphate would have an intermediate mobility between $\mathrm{CB}$ and BDP it would act as a spacer also in the peak mode shown in Figure 2, which was not observed.

Two ionic subspecies of $\mathrm{CB}$ could also cause the two plateaus. $\mathrm{CB}$ has a $\mathrm{p} K_{\mathrm{a}}$ at $7.3,{ }^{63}$ so when a small $\mathrm{pH}$ gradient exists in the chip, both $\mathrm{CB}^{3-}$ and $\mathrm{CB}^{2-}$ will be present. $\mathrm{AF} 647$ is an acidic salt, and the addition of $1 \mathrm{mM}$ in $0.1 \times \mathrm{PBS}$ is expected to result in a sample $\mathrm{pH}$ of approximately 6.9, causing a $\mathrm{pH}$ difference between the buffer in the anodic reservoir (0.1 $\times$ PBS, $\mathrm{pH} 7.4)$ and the sample ( $\mathrm{pH}$ 6.9). The more mobile $\mathrm{CB}^{3-}$ could then form the right-hand peak in Figure 3c, with the second and less bright band of blue corresponding to the "slower" $\mathrm{CB}^{2-}(3.382$ $\times 10^{-8} \mathrm{~m}^{2} \mathrm{~V}^{-1} \mathrm{~s}^{-1}$ ).

We could experimentally attempt a local quantification of $\mathrm{pH}$, but this would not be trivial. Typical $\mathrm{pH}$ quantification methods use the different charge state of reporter (fluorescent or colorimetric) molecules at various $\mathrm{pH}$. Different charge states however result in different electrophoretic mobilities which will result in different focusing location; hence, the spatial information on the $\mathrm{pH}$ is lost. In addition, since our method is also concentrating the analytes (including $\mathrm{pH}$ reporter molecules) the determination of $\mathrm{pH}$ based on the fluorescent/ color intensity is not trivial. Moreover, the influence of processes in the depletion zone itself on the local $\mathrm{pH}$ cannot be neglected. To our current knowledge, two reported works investigated the $\mathrm{pH}$ in the depletion zone in ICPF namely Mogi et al. ${ }^{64}$ and Kim et al. ${ }^{65}$ and both of them reported a reduction of $\mathrm{pH}$ at the anodic side of a cation exchange membrane such as Nafion.

In Figure 4 the evolution of the focusing process can be seen. The analytes move with a constant velocity along the $x$ direction due to the PDF. Once the analytes enter the separation chamber, they will start moving in the $y$ direction toward their focusing location by a combination of convective flow (EOF) opposed by electrophoresis (Figure 4i. As the analytes flow in the $x$ direction, they are moving continuously toward their focusing location (Figure 4i-iii). Once they form their plateaus the concentration profiles remain constant until they get extracted (Figure 4iii,iv,vi). A video of the process can be found in the SI.

Electrophoretic Spacers. Analytes which are focused in their plateau mode can be used as electrophoretic spacers to improve the resolution of analytes in peak mode. In Figure 5, CB and AF647 (spiked in $70 \mu \mathrm{M}$ to the bulk solution) are in peak mode, and BDP (spiked in $1 \mathrm{mM}$ to the bulk solution) is in plateau mode. Since the electrophoretic mobility of BDP lies in between AF647 and CB, it acts as a spacer, improving the separation resolution of the two analytes of interest (in this case

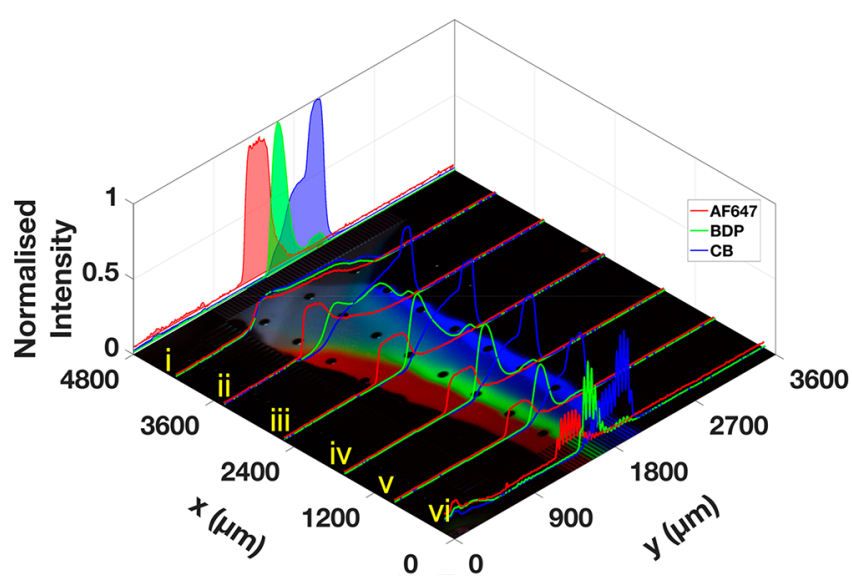

Figure 4. Evolution of concentration profiles along the $x$ direction. The profile projected on the $y-z$ plane corresponds at the location just before the extraction channels $(y=300 \mu \mathrm{m})$.

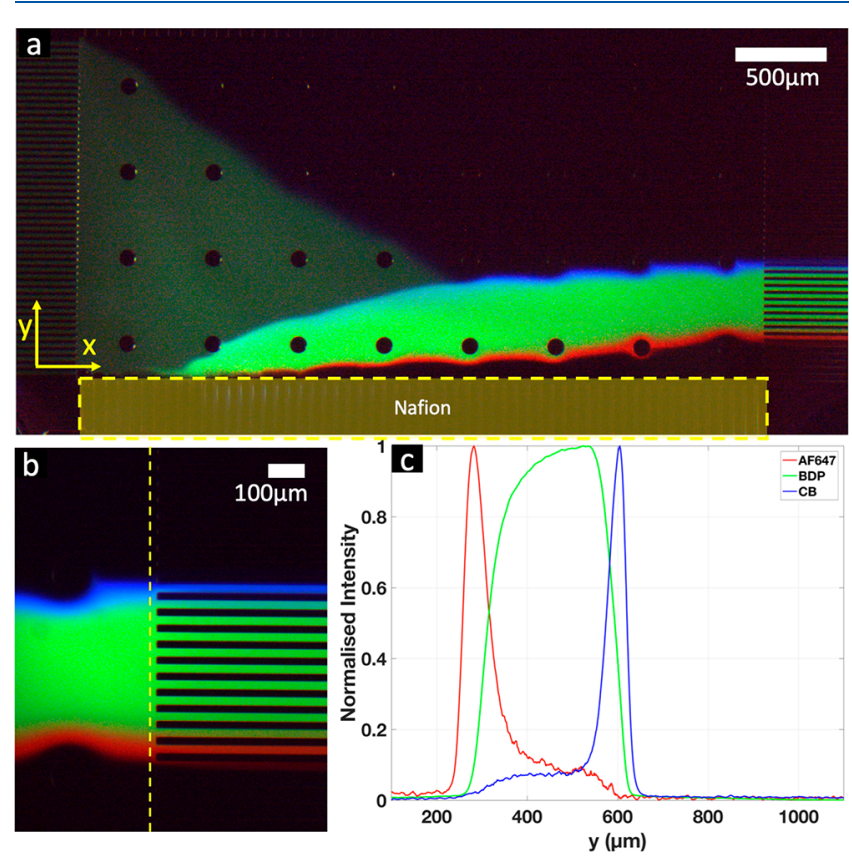

Figure 5. (a) Fluorescent microscopy image of FF-ICPF in $0.1 \times$ PBS. Focused streams of CB, BDP and AF647 can be seen $\left(5 \mu \mathrm{L} \mathrm{min}^{-1}\right.$ at 200 $\mathrm{V})$. Nafion is marked with the yellow box. (b) Close-up fluorescent microscopy image of the extracted streams. (c) Normalized fluorescence intensity profiles of CB, BDP and AF647 (along the yellow line of image $\mathrm{b}$ with $y=0$ at the Nafion membrane. BDP in plateau mode acts as an electrophoretic spacer between the $\mathrm{CB}$ and AF647. The fluorescent intensity is normalized independently for each color. Note: The contrast and color balance of microscopy images a and $\mathrm{b}$ has been altered for better visibility. The fluorescent intensity profiles have been extracted from the original images (which can be found in the SI, Figure ESI3).

$\mathrm{AF} 647$ and $\mathrm{CB})$. In this case the separation resolution between $\mathrm{AF} 647$ (red) and $\mathrm{CB}$ (blue) is $1.96\left(\sigma_{\mathrm{AF} 647}=46 \mu \mathrm{m}, \sigma_{\mathrm{CB}}=36 \mu \mathrm{m}\right.$ and the distance between peaks was $323 \mu \mathrm{m}$ as calculated from Figure 5c) compared to a resolution of 0.26 in peak mode. A higher starting concentration of BDP will result in a wider plateau further increasing the separation resolution.

Concentration Rate and Extraction Position. Both the concentration of the focused analytes and the extraction position of the streams can be tuned by the applied potential and flow rate 

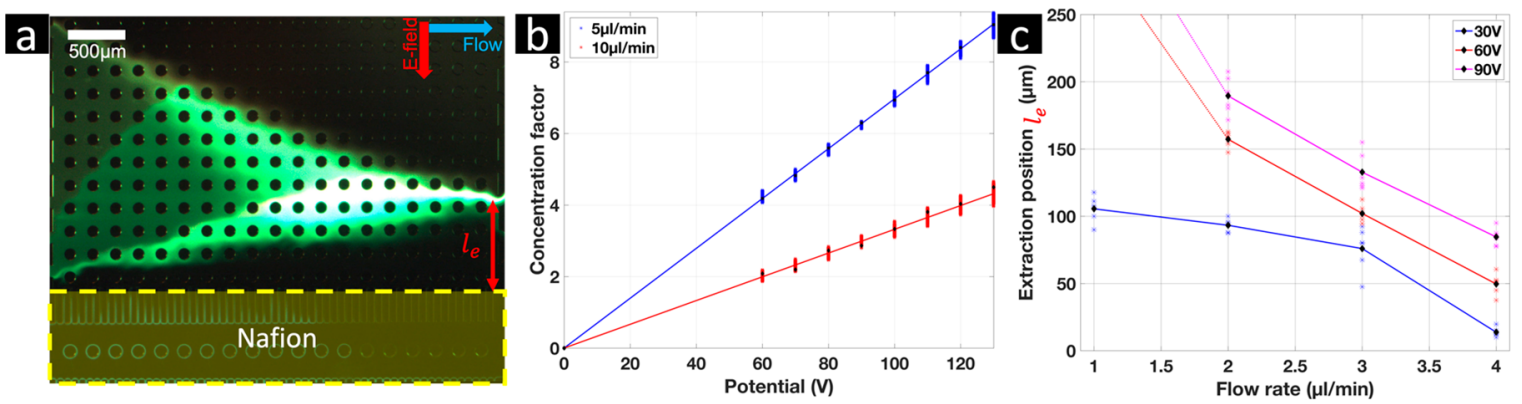

Figure 6. (a) Fluorescent microscopy image of FF-ICPF of undiluted human blood plasma ( $5 \mu \mathrm{L} \mathrm{min}{ }^{-1}$ at $\left.120 \mathrm{~V}\right)$. A highly concentrated stream of $\mathrm{BDP}$ can be seen to form. (b) Concentration factor versus potential for two flow rates. Each point $(*)$ corresponds to a different measurement of the same experiment at a different time over a duration of $100 \mathrm{~s}$ to demonstrate the stability of the system. The lines show a linear fit with $R^{2}$ of 0.9999 and 0.9934 and a slope of 0.0697 and $0.0337 \mathrm{~V}^{-1}$ for 5 and $10 \mu \mathrm{L} \mathrm{min}^{-1}$, respectively. (c) Extraction position as distance from Nafion membrane $\left(l_{\mathrm{e}}\right.$ in panel a) versus flow rate for different applied potentials. As extraction position the location of the maximum intensity of BDP was chosen. Each point $(*)$ corresponds to a separate experiment with the same device and the lines connect the average values. For a flow rate of $1 \mu \mathrm{L} \mathrm{min}{ }^{-1}$, the extraction positions of 60 and $90 \mathrm{~V}$ were outside the observation window.

of the sample. We characterized the influence of both using undiluted human blood plasma spiked with a fluorescent analyte $(100 \mu \mathrm{M}$ BDP $)$. The results are shown in Figure 6 and a video can be found in the SI. Since the analytes focus at the position in the electric field gradient where the convective and electrophoretic velocities cancel in the $y$ direction and where an electric field gradient exists between the depletion zone and the bulk, we can control the extraction location of our focused streams of analytes in the $y$ direction by controlling the size of the depletion zone. The higher the applied potential the wider the depletion zone and the further away from the Nafion the analytes will focus (Figure 6c). In addition, the depletion zone grows over distance ( $x$ direction) as the liquid flows from left to right. A faster flow rate allows less time for the depletion zone to grow, and therefore, the analytes will focus closer to the Nafion membrane (Figure 6c). Finally, the analytes are brought to their focusing location by the convective flow in the $y$ direction. The dominant contribution of convective flow in that direction is EOF. The higher the EOF, the more analytes are brought to the focusing location and the higher the concentration factor (Figure $6 \mathrm{~b}$ ). A higher flow rate (in the $x$ direction) reduces the time that the analytes spend in the separation chamber, resulting in a lower amount of analyte reaching the focusing location and a lower concentration factor. As we mentioned, the maximum concentration factor is furthermore dependent on the operation mode.

Due to the high conductivity of blood plasma, compared to the previous experiments in $0.1 \times \mathrm{PBS}$, a lower electric power (and hence throughput) could be applied before Joule heating disrupted the process. In addition, a vast number of analytes (a.o. proteins) is present in blood plasma with concentrations varying widely (albumin in the range of $\mathrm{g} \mathrm{L}^{-1}$ and some regulatory proteins in the $\mathrm{ng} \mathrm{L}^{-1}$ range); hence, some analytes will appear in plateau mode and many in peak mode. The reported extraction position is representative for the specific analyte. A different analyte with different mobility could be at a very different location due to analytes in plateau mode acting as spacers, nevertheless the location of all analytes will be shifted depending on the size of the depletion zone. The selected fluorescent analyte (BDP) is a lipophilic nonfixable polar tracer, and hence no covalent bonding to proteins is expected. If protein/BDP complexes were present we would expect them to appear as separate bands.
As shown in Figure 6, we obtained a stable separation and focusing of BDP in nondilute human blood plasma at a throughput $\left(15 \mu \mathrm{L} \mathrm{min}^{-1}\right)$ at $120 \mathrm{~V}$. A higher flow rate of $200 \mu \mathrm{L}$ $\min ^{-1}$ at $500 \mathrm{~V}$ could be achieved, while still maintaining separation and focusing but only in $10 \times$ dilute blood plasma to minimize Joule heating because of its lower electric conductivity. Nevertheless, after approximately $1 \mathrm{~min}$ the formation of bubbles could be seen in the thin channels where the current density is the highest. We believe that these bubbles were the result of local heating that reduces the solubility of gas in water. We expect that a higher stable throughput can be achieved with the use of active cooling of the device. The system was also tested for stability over a period of 20 min with no significant change in the focusing location in $0.1 \times \mathrm{PBS}$ at $150 \mathrm{~V}$. The stability time was limited by the size and buffer capacity of the reservoirs were the electrodes are emerged and Berzina et al. ${ }^{13}$ demonstrated ICPF in blood plasma stable for hours were the depletion zone prevented the biofouling of the Nafion membrane.

\section{CONCLUSIONS AND OUTLOOK}

We have demonstrated the feasibility of the continuous separation and concentration of anionic analytes via free flow ion concentration polarization focusing (FF-ICPF). The two different operation modes, namely peak and plateau mode, of the technique were investigated and demonstrated. In addition, analytes with known electrophoretic mobility in plateau mode could be used as electrophoretic spacers in order to improve the separation resolution, by a factor of 7.5 (resolution increased from 0.26 to 1.96 ). Finally, we investigated the tunability of the extraction location and concentration rate by the applied electric potential and the sample (human blood plasma) flow rate.

As a reflection we can compare our proposed method to the more popular free flow zone electrophoresis (FFZE). On the one hand FFZE requires a much easier fabrication process without the need for ion selective membranes and provides a much simpler physical system with a superior separation resolution that does not require electrophoretic spacers. In contrast, a flow focusing of the sample is required before entering the separation chamber, which makes its operation more cumbersome. More importantly, the separated streams are prone to diffusion which reduces their concentration. On the other hand, ICPF provides a simultaneous separation and 
concentration with no need for buffers or flow focusing at the cost of separation resolution.

We believe that FF-ICPF can be a promising candidate for sample preparation of biological samples. With the use of more heat conductive substrates or active cooling even higher throughputs can be achieved making it suitable for a wide variety of applications.

\section{ASSOCIATED CONTENT}

\section{st Supporting Information}

The Supporting Information is available free of charge at https://pubs.acs.org/doi/10.1021/acs.analchem.9b04526.

Demonstration of plateau mode in FF-ICPF of $100 \mu \mathrm{M}$ BDP (green) in undiluted blood plasma; actuation potential: $120 \mathrm{~V}$, flow rate: $5 \mu \mathrm{L} \min ^{-1}$ (in real time) (MP4)

Demonstration of plateau mode in FF-ICPF of $1 \mathrm{mM}$ BDP (green), $1 \mathrm{mM} \mathrm{AF647} \mathrm{(red),} \mathrm{and} 1 \mathrm{mM} \mathrm{CB}$ (blue) in $0.1 \times \mathrm{PBS}$; actuation potential: $200 \mathrm{~V}$, flow rate: $5 \mu \mathrm{L}$ $\mathrm{min}^{-1}$ (In real time) (AVI)

Details on the effect of high electric power to the stability of the system and original microscopy images (PDF)

\section{AUTHOR INFORMATION}

\section{Corresponding Author}

Vasileios A. Papadimitriou - BIOS Lab on a Chip group, MESA + Institute for Nanotechnology, Max Planck Centre for Complex Fluid Dynamics and Technical Medical Centre, University of Twente, Enschede 7500 AE, The Netherlands; 이이.org/ 0000-0001-7145-8877; Email: v.papadimitriou@utwente.nl

\section{Authors}

Loes I. Segerink - BIOS Lab on a Chip group, MESA+ Institute for Nanotechnology, Max Planck Centre for Complex Fluid Dynamics and Technical Medical Centre, University of Twente, Enschede 7500 AE, The Netherlands

Jan C. T. Eijkel - BIOS Lab on a Chip group, MESA+ Institute for Nanotechnology, Max Planck Centre for Complex Fluid Dynamics and Technical Medical Centre, University of Twente, Enschede 7500 AE, The Netherlands

Complete contact information is available at:

https://pubs.acs.org/10.1021/acs.analchem.9b04526

\section{Notes}

The authors declare no competing financial interest.

\section{ACKNOWLEDGMENTS}

This work was supported and funded by the Horizon 2020 Framework Programme of the European Union under the project H2020-PHC-634013 (PHOCNOSIS).

\section{REFERENCES}

(1) Hlushkou, D.; Perdue, R. K.; Dhopeshwarkar, R.; Crooks, R. M.; Tallarek, U. Lab Chip 2009, 9, 1903.

(2) Rickwood, D. Lab. Tech. Biochem. Mol. Biol. 1983, 11, xiii.

(3) Rodriguez-Diaz, R.; Wehr, T.; Zhu, M. Electrophoresis 1997, 18, 2134

(4) Jacroux, T.; Bottenus, D.; Rieck, B.; Ivory, C. F.; Dong, W. J. I. Electrophoresis 2014, 35, 2029-2038.

(5) Karsenty, M.; Rosenfeld, T.; Gommed, K.; Bercovici, M. Anal. Chem. 2015, 87, 388.

(6) Wang, Y. C.; Stevens, A. L.; Han, J. Anal. Chem. 2005, 77, 4293.
(7) Quist, J.; Janssen, K. G. H.; Vulto, P.; Hankemeier, T.; Van Der Linden, H. J. Anal. Chem. 2011, 83, 7910-7915.

(8) Kim, S. J.; Li, L. D.; Han, J. Langmuir 2009, 25, 7759-7765.

(9) Liu, V.; Song, Y.-A.; Han, J. Lab Chip 2010, 10, 1485.

(10) Khandurina, J.; Jacobson, S. C.; Waters, L. C.; Foote, R. S.; Ramsey, J. M. Anal. Chem. 1999, 71, 1815-1819.

(11) Ko, S. H.; et al. Lab Chip 2012, 12, 4472.

(12) Quist, J.; Vulto, P.; Van Der Linden, H.; Hankemeier, T. Anal. Chem. 2012, 84, 9065-9071.

(13) Berzina, B.; Anand, R. K. Anal. Chem. 2018, 90, 3720.

(14) Ouyang, W.; Han, J. Proc. Natl. Acad. Sci. U. S. A. 2019, 116, 16240.

(15) Phan, D. T.; Jin, L.; Wustoni, S.; Chen, C. H. Lab Chip 2018, 18, 574.

(16) Pamme, N. Lab Chip 2007, 7, 1644

(17) Turgeon, R. T.; Bowser, M. T. Anal. Bioanal. Chem. 2009, 394, 187.

(18) Janasek, D.; Schilling, M.; Franzke, J.; Manz, A. Anal. Chem. 2006, 78, 3815-3819.

(19) Poehler, E.; Herzog, C.; Lotter, C.; Pfeiffer, S. A.; Aigner, D.; Mayr, T.; Nagl, S. Analyst 2015, 140, 7496.

(20) Herzog, C.; Poehler, E.; Peretzki, A. J.; Borisov, S. M.; Aigner, D.; Mayr, T.; Nagl, S. Lab Chip 2016, 16, 1565.

(21) Kohlheyer, D.; Eijkel, J. C. T.; van den Berg, A.; Schasfoort, R. B. M. Electrophoresis 2008, 29, 977-993.

(22) Choi, J.; et al. RSC Adv. 2015, 5, 66178-66184.

(23) Chen, Y. Y.; Chiu, P. H.; Weng, C. H.; Yang, R. J. Biomicrofluidics 2016, 10, 014119.

(24) Papadimitriou, V. A.; Segerink, L. I.; Eijkel, J. C. T. Lab Chip 2019, 19, 3238-3248.

(25) Kwak, R.; Kim, S. J.; Han, J. Anal. Chem. 2011, 83, 7348.

(26) Cho, I.; Kim, W.; Kim, J.; Kim, H.-Y.; Lee, H.; Kim, S. J. Phys. Rev. Lett. 2016, 116, 254501.

(27) Kim, W.; Park, S.; Kim, K.; Kim, S. J. Lab Chip 2017, 17, 3841.

(28) Jeon, H.; Lee, H.; Kang, K. H.; Lim, G. Sci. Rep. 2013, 3, 03483.

(29) Kim, M.; Wu, L.; Kim, B.; Hung, D. T.; Han, J. Anal. Chem. 2018, 90, 872 .

(30) Kim, S. J.; Ko, S. H.; Kang, K. H.; Han, J. Nat. Nanotechnol. 2010, 5, 297.

(31) Ribeiro-Samy, S.; Oliveira, M. I.; Pereira-Veiga, T.; MuineloRomay, L.; Carvalho, S.; Gaspar, J.; Freitas, P. P.; Lopez-Lopez, R.; Costa, C.; Dieguez, L. Sci. Rep. 2019, 9, 44401-1.

(32) Varillas, J. I.; Zhang, J.; Chen, K.; Barnes, I. I.; Liu, C.; George, T. J.; Fan, Z. H. Theranostics 2019, 9, 1417.

(33) Le, V. S. Recent Results Cancer Res. 2013, 194, 43-75.

(34) Huang, Y.; Joo, S.; Duhon, M.; Heller, M.; Wallace, B.; Xu, X. Anal. Chem. 2002, 74, 3362.

(35) Heineck, D. P.; Lewis, J. M.; Heller, M. J. Electrophoresis 2017, 38, 1475

(36) Yang, J.; Selvaganapathy, P. R.; Gould, T. J.; Dwivedi, D. J.; Liu, D.; Fox-Robichaud, A. E.; Liaw, P. C. Lab Chip 2015, 15, 3925.

(37) Janasek, D.; Schilling, M.; Manz, A.; Franzke, J. Lab Chip 2006, 6, 710.

(38) Sonker, M.; Sahore, V.; Woolley, A. T. Anal. Chim. Acta 2017, 986, 1.

(39) Pu, Q.; Yun, J.; Temkin, H.; Liu, S. Nano Lett. 2004, 4, 10991103.

(40) Kim, S. J.; Wang, Y. C.; Lee, J. H.; Jang, H.; Han, J. Phys. Rev. Lett. 2007, 99, 044501.

(41) Ouyang, W.; Ye, X.; Li, Z.; Han, J. Nanoscale 2018, 10, 15187.

(42) Gong, L.; Ouyang, W.; Li, Z.; Han, J. J. Membr. Sci. 2018, 556, 34.

(43) Herr, A. E.; Molho, J. I.; Santiago, J. G.; Mungal, M. G.; Kenny, T. W.; Garguilo, M. G. Anal. Chem. 2000, 72, 1053.

(44) Benneker, A. M.; Wood, J. A.; Tsai, P. A.; Lammertink, R. G. H. Sci. Rep. 2016, 6, 37236.

(45) Kwak, R.; Pham, V. S.; Han, J. J. Fluid Mech. 2017, 813, 799.

(46) Pham, S. V. Phys. Rev. E: Stat. Phys., Plasmas, Fluids, Relat. Interdiscip. Top. 2016, 93, 033114. 
(47) De Valença, J. C.; Wagterveld, R. M.; Lammertink, R. G. H.; Tsai, P. A. Phys. Rev. E - Stat. Nonlinear, Soft Matter Phys. 2015, 92, 031003.

(48) de Valenca, J.; Jogi, M.; Wagterveld, R. M.; Karatay, E.; Wood, J. A.; Lammertink, R. G. H. Langmuir 2018, 34, 2455.

(49) Kim, K.; Kim, W.; Lee, H.; Kim, S. J. Nanoscale 2017, 9, 3466.

(50) Rubin, S.; Schwartz, O.; Bercovici, M. Phys. Fluids 2014, 26, 012001.

(51) Giddings, J. C. Unified Separation Science; John Wiley \& Sons: New York, 1991.

(52) MacInnes, D. A.; Longsworth, L. G. Chem. Rev. 1932, 11, 171230.

(53) Tiselius, A. Trans. Faraday Soc. 1937, 33, 524-531.

(54) Cheow, L. F.; Sarkar, A.; Kolitz, S.; Lauffenburger, D.; Han, J. Anal. Chem. 2014, 86, 7455 .

(55) Kohlrausch, R. Ann. Phys. 1854, 167, 179-214.

(56) Hruška, V.; Gaš, B. Electrophoresis 2007, $28,3$.

(57) Lee, J. H.; Song, Y. A.; Han, J. Lab Chip 2008, 8, 596.

(58) Weibel, D. B.; DiLuzio, W. R.; Whitesides, G. M. Nat. Rev. Microbiol. 2007, 5, 209-218.

(59) Papadimitriou, V. A.; Van Den Berg, A.; Eijkel, J. C. T. 3D capillary valves for versatile capillary patterning of channel walls. 20th International Conference on Miniaturized Systems for Chemistry and Life Sciences, MicroTAS 2016, 2016.

(60) Splawn, B. G.; Lytle, F. E. Anal. Bioanal. Chem. 2002, 373, 519.

(61) Evans, R.; Deng, Z.; Rogerson, A. K.; McLachlan, A. S.; Richards, J. J.; Nilsson, M.; Morris, G. A. Angew. Chem. 2013, 125, 3281.

(62) Vanysek, P. Ionic conductivity and diffusion at infinite dilution. CRC Handbook of Chemistry and Physics, 91th ed.; CRC Press: Bocca Raton, FL, 1991.

(63) Zhujun, Z.; Seitz, W. R. Anal. Chim. Acta 1984, 160, 47.

(64) Mogi, K. Micromachines 2018, 9, 167.

(65) Kim, W.; Oh, J.; Kwon, S.; Kim, K.; Kim, S. J. Lab Chip 2019, 19, 1359. 